\title{
A Deformable Model to Search Characteristic Facial Points
}

\author{
Reimer-A. Romero-H. ${ }^{1,2}$ and Francisco-J. Renero-C. ${ }^{1}$ \\ 1 Instituto de Astrofísica Óptica y Electrónica. Sta Ma. Tonantzintla, Puebla, \\ St. Luis Enrique Erro No 1, México \\ \{romeroreimer, paco\}@inaoep.mx \\ 2 Departamento de Física-Facyt, Universidad de Carabobo, Ave. Universidad, Sector \\ Bárbula, Carabobo, Venezuela
}

\begin{abstract}
A methodology is proposed to determine the positions of the characteristic facial points. The method is based on the Point Distribution Model, which estimates the most likely positions points. The search is refined with a discriminator, acting locally, around each point of the model; this discriminant model was achieved by training a Support Vector Machine with vectorized images in their histograms of oriented gradient (HoG). Then we aproximate de points by Thin Plate Spline by using a lambda parameters whose values were adjusted according to the local search errors. Models achieved performances that reached $90 \%$ in a cross-validation. Likewise, the strategy shows an error accumulated less than $10 \%$ in some characteristic points. The algorithm was evaluated with MUCT and BioID databases. This strategy would make digital morphological and anthropometric assessments to human face.
\end{abstract}

Keywords: Point Distribution Model, face landmarking, TPS.

\section{Introduction}

The landmarking problem have importance in face recognition, as well as in other areas biometrics. In the facial anthropometry, highlights determine measurements and proportions in the human face, which transcends in the area of cosmetic and facial surgery, and physiatry [1. A quantitative assessment of facial palsy could be of great value to the health care professional. One way to accomplish this task is by validating digital tools that would achieve accurate measurements in patient outcomes.

The main challenge of landmarking job underlay on factors like illumination, occlusion, pose and expression. For this, some aproximations have been made to improve the landmark performance. The active appearance model (AAM) and the active shape model (ASM), emerge like alternative for landmarking, both aproximations represents the face by a shape model containing the facial landmark and variability model [2].

Cristinancce and Cootes [3] apply a model of local constraint that consists on two sub-models: a shape-constrained face (PCA) and patch models, it produces

E. Bayro-Corrochano and E. Hancock (Eds.): CIARP 2014, LNCS 8827, pp. 933939 2014.

(C) Springer International Publishing Switzerland 2014 
iteratively landmark templates and applies a shape-constrained search technique, their results show a improvement respect to ASM and AAM. Albiol et al [4, detect eyes in two steps, first apply a classifier Ada Bost for selecting candidate regions in the eyes and then refined it with a support vector machine (SVM) with a descriptors using histogram of gradient oriented (HoG), the algorithm have good accuracy over the art state. And Sang Yang [5], also trained support vector machine to detect eyes, their algorithm performs the search in two stages, first a heavy neighboring blocks is performed to distinguish between the candidate areas to be one eye, then fine stage applies a SVM on these candidates to complete the search, the algorithm still performs well when required, in the first stage of the tour around the face.

This paper describe an algorithm that finds fourteen characteristic points of the human face: pupils, inner and outer eyes corner, outer and inner eye brow, left and right mouth corner, left and right nose alares. The algorithm is based on the idea of eigenvectors reconstructing the face shape, i.e., with a set training landmarked images, a principal component analysis (PCA) of the shape face models the variability of each point and begins the points search. Then, the search is refined by a support vector machine (SVM), which vector features are constructed with histogram of oriented gradient (HOG). First of all, the face recognition is done by the Viola-Jones method, which also escale and translate over a human shape model. Thus, the proposed searching algorithm, of the characteristic human face, look up for the best SVM score at the different points and by means of while loop. Finally, the TPS approximate the SVM results to the face shape model (PDM). The algorithm was proved using two public databases: BioID [6] and MUCT [7]. The patch model performance reached $90 \%$ in a cross-validation. Likewise, the strategy shows a error accumulated less than $10 \%$ in some characteristic points.

\section{SVM and Histogram of Oriented Gradient}

The SVM have been widely used for learning clasification and regression [8] 9]. It is is a discriminative method that can separate classes of objects with $n$ dimentional features with excellent generalization capability, which makes it very useful in the task of image recognition. Model requires the input of a training set $\left\{\left(\mathbf{x}_{1}, y_{1}\right),\left(\mathbf{x}_{2}, y_{2}\right), \ldots,\left(\mathbf{x}_{n}, y_{n}\right)\right\}$, with $\mathbf{x}_{i} \in \Re^{n}$ and $y_{i} \in\{+1,-1\}$ denoting the class to which the point belongs. So the SVM clasification function take the form $f(\mathbf{x})=\mathbf{w} \cdot \mathbf{x}-b$, where $\mathbf{w}$ is the weight vector and $b$ is the bias, which is computed by SVM in the training process. A correct classify ocurrs if $f(\mathbf{x})$ return a positive number for positive data points and negative number for negative data points.

In this way the image should be preprocessed to obtain a vector $\mathbf{x}$ that describes it. A descriptor frecuently used is the Histrogram of Oriented Gradient (HoG) because it offers the characteristics to avoid the extrinsic factors like illumination. The HOG descriptors are invariant to image rotation, they have been used for various tasks in the area of visual computing such as pedestrian detection [10] 11] and face recognition [12. 
The descriptor takes a local statistic of the orientations of the gradients of the image around a point. The basic idea is that the appearance of objects in an image can be characterized by the distribution of local intensity gradients or the direction of the edges, even without precise knowledge of the corresponding gradient or edge position. The strategy begins by dividing the region into cells equally spaced from each cell of a histogram of the gradient direction and orientations of the edges on all constructed the pixels of the cell. The complete histogram, with each cell represents the point descriptor. The image of a pupil at a window of $16 \times 16$ pixels is shown in figure 13 , also the magnitudes of the gradients in the Figure 10. A descriptor $4 \times 4$ pixels per cell and 9 bins is used to trace the image of the pupil, resulting in a vector of 16 descriptors, each one with a histogram of 9 bins, i.e., a vector whose dimesion is 144; the first four descriptors are shown in figure 1.

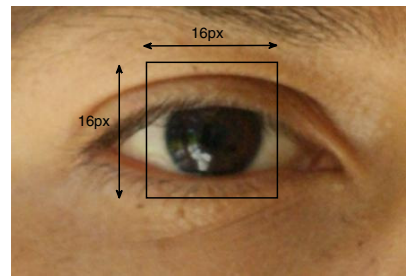

(a)

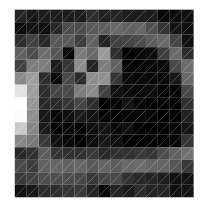

(b)

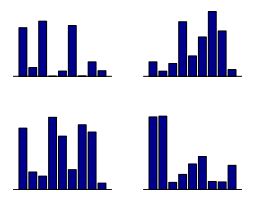

(c)

Fig. 1. HOG descriptor. (a) Eye image, (b) pupil gradient image, (c) HOG's first four cell.

\section{Thin Plate Spline Aproximation}

Thin plate spline is widely known for its use in image registration problem 13 14] 15], i.e., it is applied in combining image of the same subject from diferent modalities. However, Spriegel et. al [16], assert that in real applications the positions of the landmarks can only be determined approximately.

The scheme of TPS begin by considering to $\mathbf{p}_{i}=\left(x_{i}, y_{i}\right)$ and $\mathbf{q}_{i}=\left(x_{j}, y_{j}\right), i=$ $j=1, \ldots, n$ represent two sets of corresponding landmarks in the reference and target images respectively, then, the TPS interpolation $f(x, y)=[f]$ minimizes the bending energy:

$$
I_{f}=\iint_{\Re^{2}}\left(f_{x x}^{2}+2 f_{x y}^{2}+f_{y y}^{2}\right) d x d y
$$

and has the form:

$$
f(x, y)=a_{1}+a_{2} x+a_{3} y+\sum_{i=1}^{n} b_{i} U\left(\left(x_{i}, y_{i}\right)-(x, y)\right)
$$


The affine parameters $\mathbf{a}=\left(a_{1}, a_{2}, a_{3}\right)^{T}$ and the TPS parameters $\mathbf{b}=$ $\left(b_{1}, \ldots, b_{n}\right)^{T}$ of the transformation can be computed through the following system of system linear equations [17]:

$$
\begin{aligned}
& \left(\mathbf{K}+\lambda \mathbf{W}^{-1}\right) \mathbf{b}+\mathbf{P} \mathbf{a}=\mathbf{v} \\
& \mathbf{P}^{T} \mathbf{b}=0
\end{aligned}
$$

where $\mathbf{v}$ is the column vector of one component of the coordinates of the target points $\mathbf{q}_{i}, \mathrm{y} K_{i j}=U\left(\mathbf{p}_{i}, \mathbf{p}_{j}\right)$. Furthermore, the scalar weights representing isotropic landmark localization error are resume in the $\mathbf{W}$ matrix:

$$
\mathbf{W}=\operatorname{diag}\left\{1 / \sigma_{1}, \ldots, 1 / \sigma_{n}\right\}
$$

Finally, about the $\lambda$ parameter, this determine the relative weight between the aproximation behaviour and the smoothness of the transformation. We use two separate TPS function $f(x, y)=\left[f_{x}(x, y), f_{y}(x, y)\right]$ to map each point $\mathbf{p}_{i}$ to its homolog $\mathbf{q}_{i}$, named a pair of thin plate spline.

\section{Metodology: The Deformable Model for Searching Face Points}

Figure 2 shows the strategy followed in this work: a trainig and a serching phase. The first one builds the shape model and the patch models. We use the MUCT databases to training fase, this database have a file annotations with 76 landmarks, we used fourteen of them: pupils (pu), inner corners of eyes (in), outer cornes of eye, inner eye brow, outer eye brow, mouth cornes (ch), nose corners (al).

Because we pretend to use this to medical applications, we prefer to have a controlled enviroment, so we care about the images used, only front and noglasses image faces. With PCA on the marks (from file annotations) we find the mean model and its variability; an analysis to reduce the number of eigenvalues was made. Fourteen patch models was training with SVM, a polynomial $(n=2)$ kernel and C-soft parameter (=1) show the best performance (over the $90 \%$ ), also the patch images was vectorized with Histrogram of Oriented Gradient in cells of $4 \times 4$ pixels y 9 bins.

Searching phase begin with a Viola-Jones algorithm to capture the face image and their dimensions, next it sets the escale and translations over the shape model. A $E_{\text {min }}$ reference energy is calculated between the mean shape points and smaller deformation by equation (2), this value controls the interactions of the while loop to search all the points. At each point, the search algorithm is move in the direction of principal variability, and find the SVM best score.

We are using PCA points like reference and SVM results like target, in this way the TPS would be a interpolation $(\lambda \rightarrow 0)$ when SVM results are good and afin transformation $(\lambda \rightarrow \infty)$ when the SVM don't find a good score (and high error). 


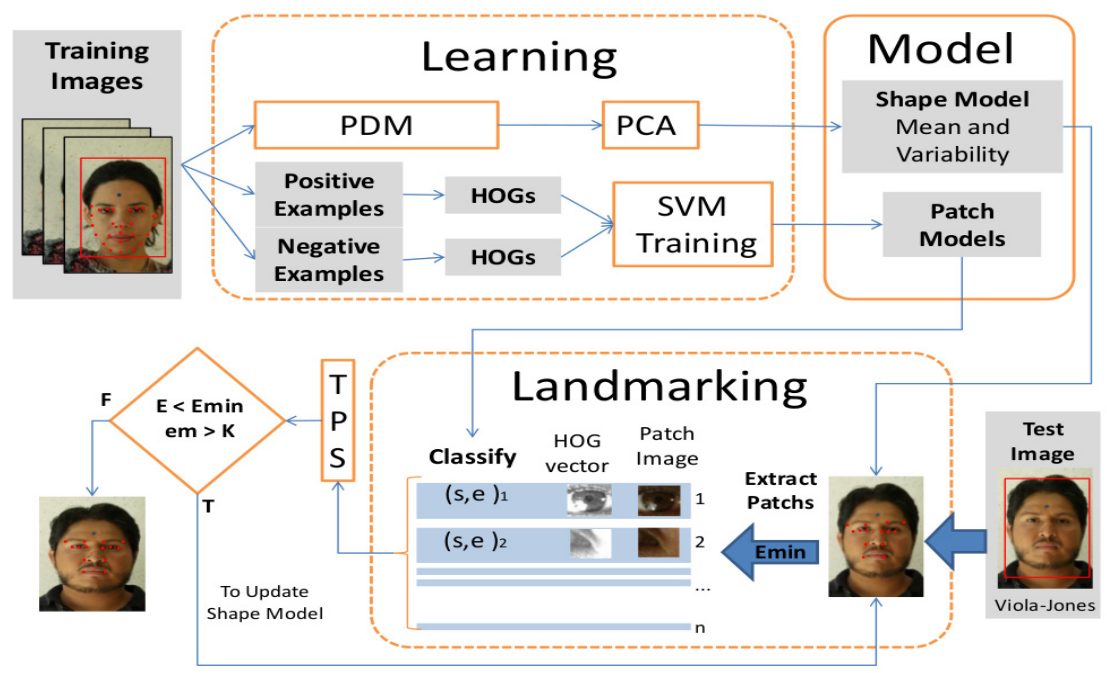

Fig. 2. Scheme of the strategy to contruct the deformable model

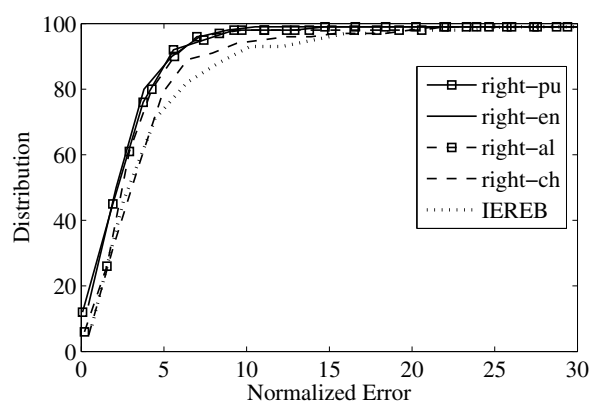

(a)

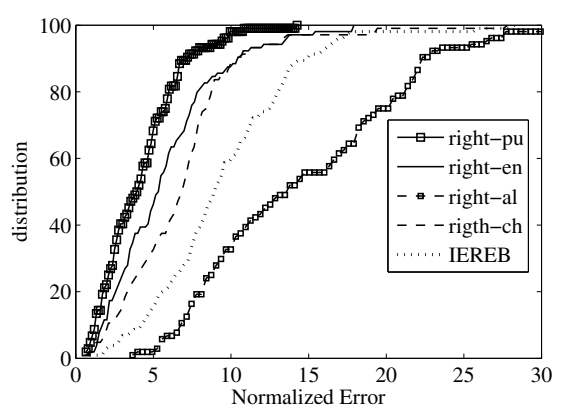

(b)

Fig. 3. Cumulative histograms over the MUCT database (a), and the BioID database (b)
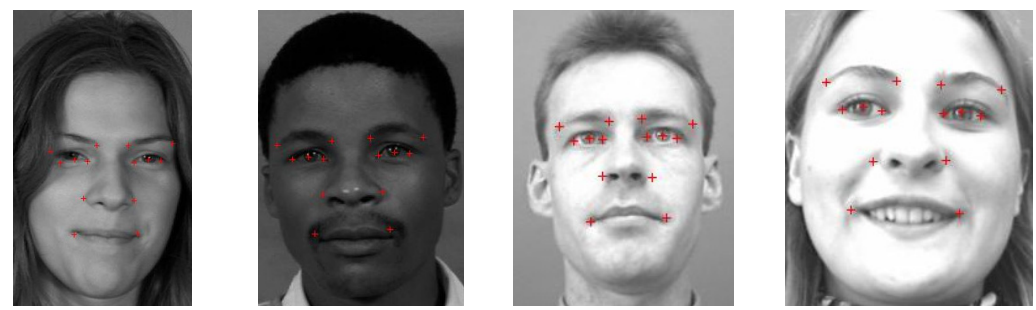

Fig. 4. Some graphical results over the databases analyzed 
We prove the algorithm with 104 images of BioID database and 100 images of MUCT database under a scheme of cros-correlation.

\section{$5 \quad$ Results}

Our results are summarized in the cumulative histograms shown in the figures 3a y 3b to databases analyzed. It can shown that this performace evaluation is comparable with the state of the art. However, the inner end of right eye brow (IEREB) of BioID databases show a deficient performace, because we are comparing the alar position detected by the algorithm with the nostril position labeled by the database. Some graphical results are shown in the figure 4.

\section{Conclusions}

In this work we implemented an algorithm to find fourteen landmark over the human face. The algorithm is based on the Point Distribution Model. We trained a shape model with positions of the landmark of a database, moreover the path models were trained with a kernel SVM. The search uses both models to find the points through a local searching oriented by a distribution points and ther variability. An aproximate TPS adjust the SVM results to model shape acoording the points variance. The evaluations on the databases analyzed shown a good agrement with the state of art.

\section{References}

[1] Preddy, V.R. (ed.): Handbook of Anthropometry. Springer (2012)

[2] Nixon, M.S., Aguado, A.S.: Feature Extraction \& Image Processing for Computer Vision, 2nd edn. Elseiver Academic Press (2008)

[3] Cristinacce, D., Cootes, T.: Automatic feature localisation with constrained local models. Pattern Recognition 41(10), 3054-3067 (2008)

[4] Monzo, D., Albiol, A., Sastre, J., Albiol, A.: Precise eye localization using hog descriptors. Mach. Vision Appl. 22(3), 471-480 (2011)

[5] Yan, L., Sang, X.: A robust eye detection method based on multi-neighborhood block with weight and svm on gray image (2013)

[6] Jesorsky, O., Kirchberg, K.J., Frischholz, R.W.: Robust Face Detection Using the Hausdorff Distance. In: Bigun, J., Smeraldi, F. (eds.) AVBPA 2001. LNCS, vol. 2091, pp. 90-95. Springer, Heidelberg (2001)

[7] Milborrow, S., Nicolls, F.: Locating Facial Features with an Extended Active Shape Model. In: Forsyth, D., Torr, P., Zisserman, A. (eds.) ECCV 2008, Part IV. LNCS, vol. 5305, pp. 504-513. Springer, Heidelberg (2008)

[8] Abe, S.: Support Vector Machines for Pattern Classification. In: Advances in Pattern Recognition. Springer, Heidelberg (2010)

[9] Vapnik, V.: Statistical learning theory. Wiley (1998)

[10] Raxle Wang, C.-C., Lien, J.-J.J.: AdaBoost Learning for Human Detection Based on Histograms of Oriented Gradients. In: Yagi, Y., Kang, S.B., Kweon, I.S., Zha, H. (eds.) ACCV 2007, Part I. LNCS, vol. 4843, pp. 885-895. Springer, Heidelberg (2007) 
[11] Dalal, N., Triggs, B.: Histograms of oriented gradients for human detection. In: IEEE Computer Society Conference on Computer Vision and Pattern Recognition, CVPR 2005, vol. 1, pp. 886-893. IEEE (2005)

[12] Albiol, A., Monzo, D., Martin, A., Sastre, J., Albiol, A.: Face recognition using hog-ebgmebgm. Pattern Recognition Letters 29(10), 1537-1543 (2008)

[13] Lewis, J.P., Hwang, H.-J., Neumann, U., Enciso, R.: Smart point landmark distribution for thin-plate splines. vol. 5370, pp. 1236-1243 (2004)

[14] Mitra, J., Marti, R., Oliver, A., Llado, X., Vilanova, J.C., Meriaudeau, F.: A comparison of thin-plate splines with automatic correspondences and b-splines with uniform grids for multimodal prostate registration. vol. 7964, pp. 79642T79642T-8 (2011)

[15] Bookstein, F.L.: Shape and the information in medical images: A decade of the morphometric synthesis. Comput. Vis. Image Underst. 66(2), 97-118 (1997)

[16] Sprengel, R., Rohr, K., Stiehl, H.S.: Thin-plate spline approximation for image registration. In: Proceedings of the 18th Annual International Conference of the IEEE Engineering in Medicine and Biology Society, Bridging Disciplines for Biomedicine, vol. 3, pp. 1190-1191 (October 1996)

[17] Rohr, K., Stiehl, H.S., Sprengel, R., Buzug, T.M., Weese, J., Kuhn, M.H.: Landmark-based elastic registration using approximating thin-plate splines. IEEE Transactions on Medical Imaging 20(6), 526-534 (2001) 